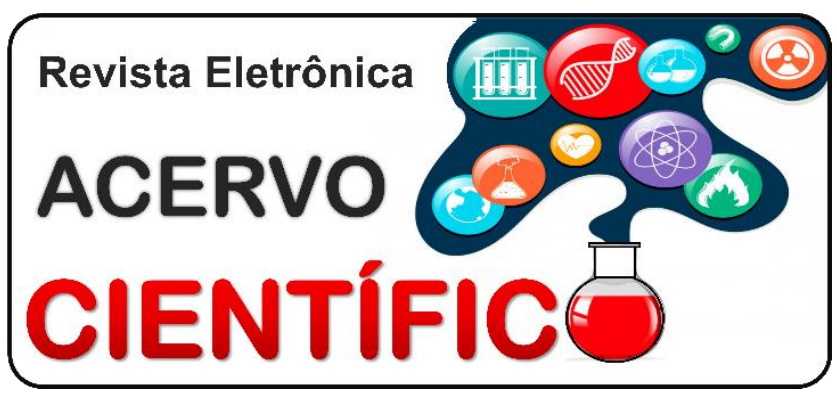

\section{REVISÃO BIBLIOGRÁFICA}

Recebido em: 10/2020

Aceito em: $11 / 2020$

Publicado em: 12/2020

\title{
Impasses psicossociais em pacientes estomizados: uma contribuição para o bem-estar desses indivíduos
}

\author{
Psychosocial impasses in ostomized patients: a contribution to the well-being of these \\ individuals
}
Impasos psicosociales en pacientes ostomizados: una contribución al bienestar de estas personas

Ingrid Hovsepian de Souza1*, Wagner Pablo Corrêa², Priscila Libman ${ }^{3}$, Leonardo Feliciano Souza ${ }^{4}$, Thalia Pimentel Caldeira Coelho ${ }^{5}$, Mariana Maia de Oliveira ${ }^{6}$, Caroline Silva de Araujo Lima ${ }^{7}$, Camila Soares Meira ${ }^{8}$, Ana Luiza Andrade Fantoni ${ }^{8}$, Débora Stefanello 9.

\begin{abstract}
Resumo: Esse artigo buscou discutir o processo de adaptação à estomia, visando principalmente os aspectos emocionais e sociais envolvidos na vida dos pacientes que possuem a bolsa coletora após passarem por esse procedimento cirúrgico, que pode ser realizado a nível de trato gastrointestinal ou em região de trato urinário, conforme a necessidade. Avaliou-se os impactos em seu meio de convívio social, como os preconceitos e estigmas sofridos, expondo como a realização dos novos cuidados indispensáveis devido a essa modificação no corpo, ou a falta dessa realização, afetará a saúde física e até mesmo psíquica dos indivíduos que necessitam conviver com um estoma, analisando as possíveis repercussões frente a essa situação. O trabalho mostra os cuidados diários dos pacientes com a bolsa coletora, observando o desconforto com o objeto, além da dedicação no cotidiano para a manutenção e higiene da mesma, gerando a necessidade de um autocuidado mais intenso. Esse processo de adaptação à convivência com a alteração corporal descrita é responsável muitas vezes pelo aparecimento de sentimentos negativos, que precisam ser revertidos em um bem-estar para esses indivíduos.
\end{abstract}

Palavras-chave: Estomia, Ajustamento emocional, Ajustamento social.

\footnotetext{
Abstract: This article sought to discuss the process of adaptation to the ostomy, focusing mainly on the emotional and social aspects involved in the lives of patients who have the collection bag after undergoing this surgical procedure, which can be performed at the gastrointestinal tract level or in the region of urinary tract,

${ }^{1}$ Universidade de Uberaba (UNIUBE), Uberaba - MG. *E-mail: ingridhovsepian1997@gmail.com

2 Faculdade de Medicina do Vale do Aço (UNIVAÇO), Ipatinga - MG.

${ }^{3}$ Fundação Técnica Souza Marques, Rio de Janeiro - RJ.

${ }^{4}$ Centro Universitário de Caratinga (UNEC), Caratinga - MG.

${ }^{5}$ Universidade José do Rosário Vellano (UNIFENAS-BH), Belo Horizonte - MG.

${ }^{6}$ Faculdade de Medicina de Petrópolis (FMP), Petrópolis - RJ,

${ }^{7}$ Faculdade Dinâmica Vale do Piranga (FAPID), Ponte Nova - MG

8 Instituto Metropolitano de Ensino Superior (IMES), Ipatinga - MG.

${ }^{9}$ Universidade Federal de Santa Maria (UFSM), Santa Maria - RS.
} 
as needed. The impacts on their social environment, such as the prejudices and stigmas suffered, were evaluated, explaining how the realization of new essential care due to this change in the body, or the lack of this realization, will affect the physical and even psychic health of individuals who need to live with a stoma, analyzing the possible repercussions in the face of this situation. The work shows the daily care of patients with the collection bag, observing the discomfort with the object, in addition to the daily dedication to its maintenance and hygiene, generating the need for more intense self-care. This process of adaptation to living with the described body alteration is often responsible for the appearance of negative feelings, which need to be reversed in a well-being for these individuals.

Keywords: Ostomy, Emotional adjustment, Social adjustment.

Resumen: Este artículo buscó discutir el proceso de adaptación a la ostomía, enfocándose principalmente en los aspectos emocionales y sociales involucrados en la vida de los pacientes que tienen la bolsa recolectora luego de ser sometidos a este procedimiento quirúrgico, el cual puede realizarse a nivel del tracto gastrointestinal o en la región de tracto urinario, según sea necesario. Se evaluaron los impactos en su entorno social, como los prejuicios y estigmas sufridos, explicando cómo la realización de nuevos cuidados esenciales debido a este cambio en el cuerpo, o la falta de esta realización, afectará la salud física e incluso psíquica de las personas que necesitan convivir con un estoma, analizando las posibles repercusiones ante esta situación. El trabajo muestra el cuidado diario de los pacientes con la bolsa colectora, observando la incomodidad con el objeto, además de la dedicación diaria a su mantenimiento e higiene, generando la necesidad de un autocuidado más intenso. Este proceso de adaptación a convivir con la alteración corporal descrita suele ser responsable de la aparición de sentimientos negativos, que necesitan revertirse en bienestar para estos individuos.

Palabras clave: Estomía, Ajuste emocional, Ajuste social.

\section{INTRODUÇÃO}

A estomia é um procedimento cirúrgico que consiste na criação de uma passagem entre um órgão do trato gastrointestinal ou urinário e o meio externo, em que se acopla uma bolsa coletora para armazenamento dos fluidos e/ou produtos do metabolismo desviados de seu trajeto orgânico. Essa intervenção pode ser realizada ao nível do intestino grosso (colostomia), intestino delgado em sua porção final (ileostomia), trato urinário (urostomia) e pode ser classificada como temporária ou permanente.

A estomia é indicada quando alguma patologia, como o adenocarcinoma de cólon ou outros tumores, ou condição anatômica (malformações congênitas como as anomalias anorretais, por exemplo) impede o fluxo regular dos resíduos gerados por essas estruturas, sendo a abertura do estoma uma solução para o curso interrompido. (SOUZA MMT, et al., 2016).

$\mathrm{Na}$ adaptação à convivência com o corpo alterado e o surgimento de sentimentos negativos, como angústia, medo, tristeza e desamparo, os pacientes estomizados vivenciam mudanças, principalmente relacionadas à sua vida social, que podem acentuar seus sentimentos de insegurança e temor de rejeição. Destaca-se a dificuldade de reconhecimento em face à modificação corporal e perda da individualidade, aspectos que têm impacto direto na saúde mental (SILVA NM, et al., 2017). Diante dessa condição, os pacientes estomizados enfrentam questões relacionadas à autoestima, qualidade de vida e relações sociais, sendo necessária uma adaptação no âmbito físico e psíquico após o procedimento (FARIA FL, et al., 2018).

A estomia requer cuidados específicos, medidas de higiene e provoca alterações na rotina do paciente e em muitos casos, de seus familiares e cuidadores (MONTORO CH, et al., 2016). A adequação dessa rotina requer esforço e atenção, para que o manejo da bolsa coletora garanta bem-estar ao paciente e possa minimizar o risco de complicações. Por isso, é importante que o indivíduo receba assistência integral, com abordagem interdisciplinar e especializada, com vistas à recuperação psíquica, social e física, rumo a reabilitação e aceitação (AZEVEDO C, et al., 2015). 
Sendo assim, objetivou-se discutir o processo de adaptação à estomia, visando principalmente os aspectos emocionais e sociais envolvidos na vida dos pacientes que possuem a bolsa coletora após passarem por esse procedimento cirúrgico, que pode ser realizado a nível de trato gastrointestinal ou em região de trato urinário, conforme a necessidade.

\section{REVISÃO BIBLIOGRÁFICA}

\section{Mudanças no modo de vida do paciente}

Os estomas intestinais, em geral, são da coloração róseo-avermelhado, podem sangrar discretamente com atrito e podem eliminar fezes espontaneamente (SILVA JC, et al., 2014). As consequências das estomias incluem desde a incontinência fecal e a eliminação de gases até a constipação, que limitam a vida social. Isto porque, ao eliminar fezes espontaneamente, há tendência à incontinência e os fármacos utilizados para controlar podem causar constipação. Outro fator que contribui negativamente é o rompimento da bolsa coletora da estomia que acumula as fezes e deve ser trocada constantemente (FARIA FL, et al., 2018; SILVA JC, et al., 2014).

Pode haver alergia às pomadas usadas como barreira protetora da pele adjacente contra o atrito da bolsa e ao material da da própria bolsa, como ao látex, além de infecções e dores locais (SUN V, et al., 2019; KARABULUT HK, et al., 2014). Desse modo, os pacientes ostomizados demandam muita atenção, que deve ser instruído a respeito dos cuidados locais e com a pele periestoma (SILVA NM, et al., 2017). Além disso, a altura das estomias intestinais pode demandar ainda mais cuidados por parte do paciente. Por exemplo, estomias no intestino alto e cólon ascendente tendem a eliminar maior quantidade de fezes, além de serem mais líquidas. Isso pode agravar a qualidade de vida do paciente, necessitando de maior higiene com a bolsa (SILVA JC, et al., 2014). Uma enfermeira especializada em estomias pode contribuir para reabilitação do indivíduo estomizado em relação à sua nova condição de saúde (CARVALHO SORM, et al., 2015)

É comum após a cirurgia que necessita a confecção de uma estomia, dúvidas sobre a nova condição de vida. O paciente enfrenta questões físicas estigmatizantes, emocionais e psicossociais, que limitam a sua autonomia (GENG Z, et al., 2017). Pesquisas indicaram que os pacientes com algum familiar ou pessoa próxima que já viveu com estomia tendem a pior evolução, devido ao conhecimento prévio das dificuldades (MONTORO CH, et al., 2016).

O tempo decorrido do pós operatório também influencia na qualidade de vida, uma vez que a adaptação à bolsa é importante. Portanto, pacientes recentemente ostomizados apresentam menos qualidade. Com o decorrer do tempo, principalmente após 6 meses de cirurgia, esse cenário muda positivamente, pois os indivíduos lidam melhor com as dificuldades enfrentadas no início, além de conduzirem com maior habilidade os cuidados com a bolsa (SILVA NM, et al., 2017).

O novo orifício formado pela cirurgia causa uma mudança corporal significativa, havendo pacientes que se recusam a vê-lo em um primeiro momento. Os indivíduos com câncer intestinal que entendem a cirurgia apenas como um prolongamento do sofrimento demoram mais para aceitar e se preocupam mais em ocultar o estoma. Em contrapartida, há aqueles que aceitam facilmente as mudanças no pós operatório, como pacientes com câncer intestinal que acreditam na cura após a cirurgia e tendem a aceitar as mudanças corporais mais rápido, como uma nova forma de identidade (MONTORO CH, et al., 2016).

Além das mudanças corporais, as atividades cotidianas também sofrem modificações. Achar uma roupa que esconda o estoma pode ser difícil, o que limita as atividades sociais. O banho também é comprometido, muitos pacientes relatam que os reservam apenas para os dias de troca da bolsa.

Problemas de sono surgem, principalmente pela dificuldade de achar uma posição adequada e necessidade de esvaziamento de bolsa durante a noite. Um estudo feito com pacientes muçulmanos demonstrou que pode afetar a vida religiosa, já que as práticas religiosas diárias são prejudicadas (CENGIZ B e BAHAR Z, 2017). Em relação a condição econômica, questionários aplicados por Faria, FL et al. (2018), indicam que há impacto negativo, sendo este um contribuinte para diminuição de qualidade de vida. Isso ocorre, pois muitas vezes, o paciente é afastado, demitido ou destituído de seu trabalho devido à incontinência 
fecal e privacidade demandada para os cuidados higiênicos da bolsa. Contudo, parte dos pacientes aproveitam o novo tempo extra para maior dedicação a outras atividades de prazer, o que pode ser positivo para essa parcela dos indivíduos (FARIA FL, et al., 2018). Além disso, condições financeiras precárias podem limitar o acesso à saúde e aos cuidados adequados, atuando como fator negativo (SILVA CRDT, et al., 2017).

No estudo de Karabulut, HK, et al. (2014) foi analisado a qualidade de vida e perda da independência, identificando diferenças em relação a sexo e idade. Pacientes do sexo feminino conseguem manter melhor o autocuidado e por isso se adaptam mais rápido à estomia. Em contrapartida, pessoas do sexo masculino afirmam ter maiores dificuldades de cuidarem de si mesmo, tornando-se mais dependentes. No que se refere a idade, enquanto em indivíduos idosos são mais influenciados pela dificuldade do autocuidado e menos influenciados por fatores externos como o social, este é o que mais afeta a qualidade de vida da população jovem (KARABULUT HK, et al., 2014).

Em relação aos aspectos psicológicos, há alterações que podem acometer até $20 \%$ dos pacientes (KARABULUT HK, et al., 2014). Os quadros psicológicos mais comuns são depressão, ansiedade, angústia e insegurança com a vida social. Por isso, é indispensável a ajuda dos profissionais de saúde para reinserir o indivíduo na sociedade e não apenas ter a preocupação com alterações físicas (SILVA NM, et al., 2017). Foi demonstrado níveis aumentados de neurotrofina 3 (Neurotrophin-3-NTF 3) em pacientes ostomizados, que está relacionada à distúrbios noradrenérgicos centrais. Além disso, houve redução dos níveis de fator neurotrófico derivado do cérebro (brain-derived neurotrophic factor- BNDF), importante para neuroplasticidade. Esses fatos somados justificam laboratorialmente a depressão (BAVARESCO DV, et al., 2018).

Os pacientes relatam discriminações que sofrem ao ir à praia ou quando a bolsa rompe e consequentemente há odor fétido (SILVA JC, et al., 2014). As ideias suicidas e falta de esperança crescem significativamente no pós operatório, principalmente nos 3 primeiros meses (SARABI N, 2020). O suporte familiar e de amigos é essencial para uma melhor adaptação (CENGIZ B e BAHAR Z, 2017). Grupos de apoio, estruturalmente treinados e especializados em estomia demonstraram aumento importante na qualidade de vida dos pacientes, principalmente quando focados em aspectos psicológicos e sociais (GANJALIKHANI, MK, 2019).

Outro aspecto da vida do paciente que possui estomia é a dieta. Com auxílio de um nutricionista, devem achar um equilíbrio para diminuir as limitações impostas pela incontinência fecal, eliminação de gases e evitar a constipação. Para isso, é indispensável uma alimentação rica em fibras e com restrições a resíduos, além de evitar alimentos que aumentam a produção de gases (SILVA JC, et al., 2014).

Uma queixa frequente entre os pacientes com bolsa de estomia é a mudança na vida sexual. Os fatores que contribuem para isso são a perda de libido, dificuldade de ejaculação e diminuição da lubrificação vaginal, tanto por lesões nervosas da cirurgia quanto pôr os estigmas da bolsa. Isso pode afetar a vida afetiva do indivíduo e até levar a conflitos conjugais, contribuindo para o aumento de problemas psiquiátricos, incluindo a depressão (VERA SO, et al., 2017). A família também é afetada por esse processo com alta demanda emocional, havendo mudanças na vida de todos ao redor do indivíduo ostomizado, Por isso, é necessário uma abordagem multidisciplinar incluindo todos os familiares (FARIA FL, et al., 2018).

\section{Como autocuidado influencia na saúde mental}

O bem-estar trata-se da concepção que o indivíduo detém acerca de sua posição na vida, mediante contexto cultural e em relação aos seus objetivos, expectativas e referências. Além disso, envolve também a avaliação dos impactos físicos, psicossociais e espirituais dentro de cada individualidade, e a influência deles em relação à satisfação com a vida, bem-estar e integridade. Nesse sentido, saúde e doença têm papel primordial nesse contexto (RIBEIRO WA e ANDRADE M, 2020).

A realização da estomia tem como objetivo melhorar o estado de saúde e a qualidade de vida da pessoa. Contudo, os desafios que a formação de um estoma suscita podem ter um impacto significativo em vários aspectos da vida, sendo notório ao nível fisiológico, social e psicológico. Uma vez que não há condições que o impeça, o paciente deve ser motivado e capacitado a assumir o controle de seu próprio autocuidado e para 
isso, é necessária uma abordagem de ensino, sendo comandada pelas orientações prestadas por uma equipe multiprofissional, composta principalmente por enfermeiros que saibam realizar o acolhimento do paciente estomizado (MOTA MS, et al., 2016).

O autocuidado é composto pelo exercício de atividades que são realizadas pelo indivíduo de forma espontânea e para benefício próprio (MOTA MS, et al., 2016). Essas ações têm como objetivo conservar a vida e a saúde, sendo condutas que devem ocorrer de modo intencional e voluntário favorecendo, assim, a tomada de decisões com o objetivo de propiciar uma harmonia entre o orgânico e o bem-estar biopsicossocial.

Os cuidados profissionais e familiares são cruciais logo após a cirurgia, porém devem ser, gradativamente, transferidos ao paciente à medida que ele se recupera e manifeste segurança e capacidade para realizá-los. Dessa forma, desenvolver habilidades e aptidão para o exercício do autocuidado facilita a retomada das atividades de vida diária, contribuindo para o viver com qualidade (SILVA J, et al., 2014).

O isolamento social é uma problemática comum em pacientes com estomia, em especial quando esta é recente. Isso é gerado a partir de medos, inseguranças e constrangimentos por recear que o estoma ou a bolsa coletora fiquem evidentes e ocorra algum evento desagradável. No entanto, constatou-se que, com o passar do tempo, com a aquisição de conhecimentos e a readaptação ao habitual como um indivíduo com estomia, este pode, a partir do autocuidado, se sentir apto a voltar a frequentar ambientes que evitava por introversão (SENA RMC, et al., 2017).

A adaptação à estomia é um processo proativo em contínuo aperfeiçoamento: em primeira instância, é possível perceber que a dedicação dos pacientes está voltada para cessar a carga emocional negativa, o que reflete diretamente no grau de autossuficiência dos indivíduos (SENA RMC, et al., 2017). Entretanto, com o transcorrer do tempo, é possível notar mudanças comportamentais positivas que se deram em função do desenvolvimento do autocuidado, o que reflete diretamente na saúde mental do indivíduo estomizado. Portanto, quando o sujeito se torna capaz de convencionar técnicas para articular suas emoções e resolver seus dilemas, ocorre uma harmonia nesse processo de adaptação, e tal indivíduo torna-se capaz de efetivar o seu autocuidado, que é tão necessário à sua adequada reinserção social (MOTA MS, et al., 2016).

\section{Apoio emocional prestado pelos profissionais de saúde}

Sabe-se que as estomias podem ser utilizadas no tratamento de diversas doenças, como as neoplásicas e doenças inflamatórias intestinais. No passado, a preocupação dos profissionais de saúde era com a sobrevida desses pacientes. Com uma mudança em foco, derivada de fatores como avanço na detecção e tratamento das doenças, faz-se necessário uma maior preocupação dos médicos e demais profissionais com a qualidade de vida desses indivíduos sobreviventes e o impacto das terapias utilizadas, incluindo o uso de estomias (FERREIRA EC, et al, 2017).

É fundamental que haja o acompanhamento de profissionais especializados ao longo de todo o processo, mas é igualmente importante promover a continuidade dos cuidados na Atenção Primária à Saúde e a mobilização dos recursos comunitários existentes com o intuito de proporcionar a melhor qualidade de vida aos doentes estomizados (FONSECA M, et al., 2019).

O ensino do autocuidado deve acontecer na fase perioperatória, com intervenções no pré-operatório que visam, além da demarcação do local do estoma, o ensino do paciente e família sobre a cirurgia, suas consequências e o autocuidado. No pós operatório, o ensino do autocuidado deve ser retomado, assim como cuidados com alimentação, atividades físicas e retomada das atividades cotidianas e laborais (SILVA J, et al., 2014). A adaptação após a alta hospitalar pode ser favorecida mediante o acolhimento de suas angústias, medos e dúvidas. $O$ atendimento dos profissionais da saúde deve ir muito além de fornecer kits, cartilhas e ensino do autocuidado com a colostomia e equipamentos coletores. Além disso, espaços de discussões sobre os preconceitos e estigmas sociais podem ser difundidos na sociedade (SILVA MN, et al., 2017).

É importante ressaltar que outros profissionais de saúde, não somente o médico, assumem um papel importante na nova etapa de vida do doente estomizado e família, sendo o papel dos profissionais de enfermagem fundamental para promover o autocuidado destes pacientes (MONTORO CH, et al., 2016). A atuação desse profissional é ampla e compreende conhecimentos e habilidades para colaborar no processo 
de adaptação à estomia, atuando desde o pré-operatório e intensificando sua atuação no pós-operatório. (RIBEIRO WA, et al., 2020). Um exemplo desse cuidado é realizado na Turquia, onde os indivíduos estomizados têm recebido serviço especializado de atendimento, treinamento e aconselhamento em grupos pela equipe de enfermagem há mais de 10 anos. Essa experiência demonstra que os pacientes que já estão adaptados à condição podem oferecer contribuições importantes no cuidado dos indivíduos recentemente estomizados ao compartilhar problemas e soluções que já vivenciaram (KARABULUT HK, et al., 2014).

\section{CONSIDERAÇÕES FINAIS}

Ante o exposto, mudanças foram observadas nos aspectos sociais e emocionais dos pacientes estomizados, no que tange a adaptações a um novo estilo de vida, tornando-se evidente, portanto, a necessidade de uma reflexão sobre a organização do sistema de saúde, a fim de promover uma assistência adequada a esses pacientes integrando-os à sociedade como cidadãos. Dessa forma, é mister que os profissionais de saúde sejam devidamente capacitados para lidar com esse paciente, atendendo sua demanda emocional e física, para que, assim, se torne reduzido os danos para o indivíduo estomizado após a cirurgia.

\section{REFERÊNCIAS}

1. AZEVEDO C, et al. Intervenções de enfermagem para alta de paciente com estomia intestinal: revisão integrativa. Revista Cubana de Enfermería, 2015; 30(2): 1561-2961.

2. BAVARESCO DV, et al . Depressive symptoms and neurotrophin levels in ostomy patients. J. bras. psiquiatria, 2018; 67(3): 166-173.

3. CARVALHO SORM, et al . "With some care, we can go on": experiences of people with ostomy. Texto Contexto Enferm, 2015; 24(1): 279-287.

4. CENGIZ B, BAHAR Z. Perceived Barriers and Home Care Needs When Adapting to a Fecal Ostomy: A Phenomenological Study. J Wound Ostomy Continence Nurs, 2017;44(1):63-68.

5. FARIA FL et al. Avaliação da qualidade de vida em pacientes com estomia intestinal. Arquivos de Ciências da Saúde, 2018; 25(2): 8-14.

6. FERREIRA EC, et al. Self-esteem and health-related quality of life in ostomized patients. Revista Brasileira de Enfermagem, 2017;70(2):271-8.

7. GENG Z, et al. Quality of Life in Chinese Persons Living With an Ostomy: A Multisite Cross-sectional Study. J Wound Ostomy Continence Nurs. 2017;44(3):249-256.

8. MONTORO $\mathrm{CH}$, et al. Experiences and coping with the altered body image in digestive stoma patients. Revista LatinoAmericana de Enfermagem, 2016; 24: e2840.

9. KARABULUT HK, et al. Effects of planned group interactions on the social adaptation of individuals with an intestinal stoma: a quantitative study. Journal of Clinical Nursing, 2014;23(19-20):2800-2813.

10. GANJALIKHANI MK, el al. Studying the effect of structured ostomy care training on quality of life and anxiety of patients with permanent ostomy. Int Wound J. 2019; 16: 1383- 1390.

11. MOTA MS, et al. Autocuidado: uma estratégia para a qualidade de vida da pessoa com estomia. Investig Enferm. Imagen Desarr, 2016; 18(1): 63-78.

12. RIBEIRO WA, ANDRADE M. Perspectiva do paciente estomizado intestinal frente a implementação do autocuidado. Revista Pró-UniverSUS. 2020;11(1):06-13.

13. SARABI N. Hopelessness and suicide ideation in ostomy patients: a mixed method study. J. Coloproctology, 2020; 40 (3): 214-219.

14. SENA RMC, et al. Aspectos Emocionais do Indivíduo no Enfrentamento da Condição de Estomizado. Estima, 2017; $15(1): 43-49$.

15. SILVA CRDT, et al. Qualidade de vida de pessoas com estomias intestinais de eliminação. Acta paul. enferm, 2017; $30(2): 144-151$.

16. SILVA J, et al. Estratégias de ensino para autocuidado de estomizados intestinais. Rev Rene. 2014; 15(1):166-73.

17. SILVA NM, et al . Aspectos psicológicos de pacientes estomizados intestinais: revisão integrativa. Rev. Latino-Am. Enfermagem, 2017; 25: e2950,

18. SOUZA MMT, et al. Apoio emocional realizado por enfermeiro ao paciente ostomizado. Revista Portuguesa de Enfermagem de Saúde Mental, 2016; spe4: 49-56.

19. SUN V, et al. Cancer survivors' challenges with ostomy appliances and self-management: a qualitative analysis. Support Care Cancer. 2020; 28(4):1551-1554.

20. VERA SO, et al. Sexualidade de pacientes com estomias intestinais de eliminação Sexuality of patients with bowel elimination ostomy. Revista de Pesquisa: Cuidado é Fundamental Online, 2017; 9(2): 495-502. 